\title{
Analytical Methods:
}

Carbonate as well as gray and black shale samples were collected from surface outcrops of various cyclothems in Iowa, Misssouri, Kansas and Oklahoma. All samples were processed for conodont elements using standard preparation techniques. Conodont elements $(0.5$ to $1 \mathrm{mg})$ were dissolved in nitric acid and chemically converted into $\mathrm{Ag}_{3} \mathrm{PO}_{4}$ using a slightly modified method described by O’Neil et al. (1994). The oxygen isotope composition was measured on CO generated by reducing trisilverphosphate using a high-temperature conversion-elemental analyzer (TC-EA) connected online to a ThermoFinnigan Delta plus mass-spectrometer. Most samples were measured in triplicate. Accuracy and reproducibility was monitored by multiple analyses of trisilverphosphate prepared from NBS120c and several trisilverphosphate standards (TUI-1, TUI-2, YR-2; Vennemann et al. 2002). The average oxygen isotope composition of TUI-1, TUI-2 and YR-2 standards was 21.3, 5.5, and $13.2 \%$ V-SMOW, respectively. The mean $\delta^{18} \mathrm{O}$ value of NBS120c was $22.4 \%$ V-SMOW, which is $0.7 \%$ higher than values reported in the literature by Crowson et al. (1991) and Lécuyer et al. (1996), but relatively close to the value of $22.58 \%$ V-SMOW determined by Vennemann et al. (2002). The overall reproducibility determined by replicate analyses of trisilverphospate standards as well as replicate sample analysis was better than $\pm 0.2 \%$ o $(1 \sigma)$.

\section{References:}

Crowson, R.A., Showers, W.J., Wright, E.K., and Hoering, T.C., 1991, Preparation of phosphate samples for oxygen isotope analysis: Analytical Chemistry, v. 63, p. 2397-2400.

Lécuyer, C., Grandjean, P., and Emig, C.C., 1996, Determination of oxygen isotope fractionation between water \& phosphate from living lingulides: Potential application to palaeoenvironmental studies:. Palaeogeography, Palaeoclimatology, Palaeoecology, v. 126, p. 101-108.

O'Neil, J.R., Roe, J.L., Reinhard, E., and Blake, R.E., 1994, A rapid and precise method of oxygen isotope analysis of biogenic phosphate: Israelian Journal of Earth Sciences, v. 43, p. 203-212.

Vennemann, T.W., Fricke, H.C., Blake, R.E., O’Neil, J.R. and Colman, A., 2002, Oxygen isotope analysis of phosphates: a comparison of techniques for analysis for $\mathrm{Ag}_{3} \mathrm{PO}_{4}$ : Chemical Geology, v. 185 , p. $321-336$. 
TABLE DR 1. Oxygen isotope ratios of conodont apatite from the Pennsylvanian of USMidcontinent. IA - Iowa, KS - Kansas,. NE - Nebraska, MO - Missouri, OK - Oklahoma, Sh - shale, Ls - limestone. Sample code A to D correspond to locations given in Heckel et al. 1999. Letters in brackets indicate analysis of elements of specific genera: S - Streptognathodus sp., I - Idiognathodus sp., H - Hindeodus sp., G - Gondolella sp.

\begin{tabular}{|c|c|c|c|c|c|}
\hline Sample & Age & Formation & Member & Location & $\begin{array}{c}\delta^{18} \mathrm{O} \\
\text { (\%o V-SMOW) }\end{array}$ \\
\hline C9-III & Virgilian & Topeka Ls. & Coal Creek Ls. & Chautauqua Co. , KS & 21.0 \\
\hline C9-I & Virgilian & Topeka Ls. & Dubois Ls. & Chautauqua Co., KS & 20.6 \\
\hline C7-IV & Virgilian & Deer Creek Ls. & Ervine Creek Ls. & Chautauqua Co. , KS & 20.5 \\
\hline C7-III & Virgilian & Deer Creek Ls. & Larsh Burroak Sh. & Chautauqua Co., KS & 19.5 \\
\hline C7-II & Virgilian & Deer Creek Ls. & Larsh Burroak Sh. & Chautauqua Co. , KS & 19.5 \\
\hline C7-I & Virgilian & Deer Creek Ls. & Larsh Burroak Sh. & Chautauqua Co., KS & 19.9 \\
\hline C7-V & Virgilian & Deer Creek Ls. & Rock Bluff Ls. & Chautauqua Co., KS & 20.2 \\
\hline C5-II & Virgilian & Lecompton Ls. & Queen Hill Sh. & Chautauqua Co., KS & 20.5 \\
\hline C5-I & Virgilian & Lecompton Ls. & Queen Hill Sh. & Chautauqua Co. , KS & 20.0 \\
\hline P-14-1 (S+I) & Virgilian & Oread Ls. & Plattsmouth Ls. & Cass Co., NE & 21.1 \\
\hline$P-14-2 / 3$ & Virgilian & Oread Ls. & Plattsmouth Ls. & Cass Co., NE & 21.1 \\
\hline$P-13-5$ & Virgilian & Oread Ls. & Plattsmouth Ls. & Cass Co., NE & 21.8 \\
\hline P-13-4 & Virgilian & Oread Ls. & Plattsmouth Ls. & Andrew County, MO & 21.9 \\
\hline P-11-1 & Virgilian & Oread Ls. & Plattsmouth Ls. & Leavenworth Co., KS & 21.6 \\
\hline P-10-3 & Virgilian & Oread Ls. & Plattsmouth Ls. & Leavenworth Co., KS & 21.5 \\
\hline$P-7-3$ & Virgilian & Oread Ls. & Plattsmouth Ls. & Greenwood Co., KS & 20.8 \\
\hline P-7-2 & Virgilian & Oread Ls. & Plattsmouth Ls. & Greenwood Co., KS & 20.7 \\
\hline$P-2-2 b$ & Virgilian & Oread Ls. & Plattsmouth Ls. & Chautauqua Co., KS & 21.0 \\
\hline$P-2-1 a+b$ & Virgilian & Oread Ls. & Plattsmouth Ls. & Chautauqua Co. , KS & 20.8 \\
\hline P-3-1 & Virgilian & Oread Ls. & Plattsmouth Ls. & Chautauqua Co., KS & 20.6 \\
\hline P-5-1 & Virgilian & Oread Ls. & Plattsmouth Ls. & Osage Co. OK & 20.4 \\
\hline$P-4-4$ & Virgilian & Oread Ls. & Plattsmouth Ls. & Coffey Co., KS & 21.6 \\
\hline$P-4-2$ & Virgilian & Oread Ls. & Plattsmouth Ls. & Coffey Co., KS & 21.4 \\
\hline He-14-1 (H) & Virgilian & Oread Ls. & Heebner Sh. & Cass Co., NE & 20.3 \\
\hline He-14-1 (S) & Virgilian & Oread Ls. & Heebner Sh. & Cass Co., NE & 20.4 \\
\hline He-14-1 (S+I) & Virgilian & Oread Ls. & Heebner Sh. & Cass Co., NE & 20.3 \\
\hline He-13-3 & Virgilian & Oread Ls. & Heebner Sh. & Andrew Co., MO & 21.2 \\
\hline $\mathrm{He}-13-2 \mathrm{a}$ & Virgilian & Oread Ls. & Heebner Sh. & Andrew Co., MO & 20.7 \\
\hline He-13-1 & Virgilian & Oread Ls. & Heebner Sh. & Andrew Co., MO & 20.3 \\
\hline He-11-2 & Virgilian & Oread Ls. & Heebner Sh. & Buchanan Co., MO & 20.7 \\
\hline He-11-1 & Virgilian & Oread Ls. & Heebner Sh. & Buchanan Co., MO & 20.3 \\
\hline He-10-2 & Virgilian & Oread Ls. & Heebner Sh. & Leavenworth Co. KS & 21.1 \\
\hline He-10-1 & Virgilian & Oread Ls. & Heebner Sh. & Leavenworth Co. KS & 20.2 \\
\hline $\mathrm{He}-3-4$ & Virgilian & Oread Ls. & Heebner Sh. & Coffey Co., KS & 20.7 \\
\hline $\mathrm{He}-3-1$ & Virgilian & Oread Ls. & Heebner Sh. & Coffey Co., KS & 19.9 \\
\hline He-3-1 & Virgilian & Oread Ls. & Heebner Sh. & Coffey Co., KS & 20.2 \\
\hline $\mathrm{He}-5-2$ & Virgilian & Oread Ls. & Heebner Sh. & Greenwood Co., KS & 20.3 \\
\hline $\mathrm{He}-5-1$ & Virgilian & Oread Ls. & Heebner Sh. & Greenwood Co., KS & 19.7 \\
\hline $\mathrm{He}-8-2$ & Virgilian & Oread Ls. & Heebner Sh. & Elk Co. KS & 20.8 \\
\hline He-8-1 & Virgilian & Oread Ls. & Heebner Sh. & Elk Co. KS & 19.7 \\
\hline C4-IV & Virgilian & Oread Ls. & Heebner Sh. & Chautauqua Co., KS & 21.1 \\
\hline C4-II & Virgilian & Oread Ls. & Heebner Sh. & Chautauqua Co., KS & 19.6 \\
\hline $\mathrm{He}-2-3 b$ & Virgilian & Oread Ls. & Heebner Sh. & Chautauqua Co., KS & 21.0 \\
\hline $\mathrm{He}-2-3 \mathrm{a}$ & Virgilian & Oread Ls. & Heebner Sh. & Chautauqua Co., KS & 20.5 \\
\hline
\end{tabular}




\begin{tabular}{|c|c|c|c|c|c|}
\hline $\mathrm{He}-2-2(\mathrm{~S})$ & Virgilian & Oread Ls. & Heebner Sh. & Chautauqua Co., KS & 20.0 \\
\hline $\mathrm{He}-2-2(\mathrm{H})$ & Virgilian & Oread Ls. & Heebner Sh. & Chautauqua Co., KS & 20.0 \\
\hline $\mathrm{He}-2-2 \mathrm{~b}$ & Virgilian & Oread Ls. & Heebner Sh. & Chautauqua Co., KS & 20.1 \\
\hline $\mathrm{He}-2-2 \mathrm{a}(\mathrm{H})$ & Virgilian & Oread Ls. & Heebner Sh. & Chautauqua Co., KS & 19.9 \\
\hline $\mathrm{He}-2-2 \mathrm{a}$ & Virgilian & Oread Ls. & Heebner Sh. & Chautauqua Co., KS & 19.8 \\
\hline $\mathrm{He}-2-1(\mathrm{~S})$ & Virgilian & Oread Ls. & Heebner Sh. & Chautauqua Co., KS & 20.1 \\
\hline $\mathrm{He}-2-1(\mathrm{H})$ & Virgilian & Oread Ls. & Heebner Sh. & Chautauqua Co., KS & 19.7 \\
\hline $\mathrm{He}-4-2$ & Virgilian & Oread Ls. & Heebner Sh. & Osage Co., OK & 20.1 \\
\hline He-6 rec & Virgilian & Oread Ls. & Heebner Sh. & Osage Co., OK & 20.1 \\
\hline $\mathrm{He}-7$ rec & Virgilian & Oread Ls. & Heebner Sh. & Osage Co., OK & 20.1 \\
\hline He-7-1 & Virgilian & Oread Ls. & Heebner Sh. & Osage Co., OK & 20.7 \\
\hline He 9-2 & Virgilian & Oread Ls. & Heebner Sh. & Osage Co., OK & 20.9 \\
\hline D1 V & Virgilian & Cass Ls. & Little Pawnee Sh. & Woodson Co., KS & 19.9 \\
\hline A8-III & Virgilian & Cass Ls. & Little Pawnee Sh. & Leavenworth Co. KS & 21.2 \\
\hline C3-IV & Virgilian & Cass Ls. & Little Pawnee Sh. & Chautauqua Co., KS & 19.9 \\
\hline C3-II & Virgilian & Cass Ls. & Little Pawnee Sh. & Chautauqua Co., KS & 20.8 \\
\hline A8-VI & Virgilian & Cass Ls. & Haskell Ls. & Leavenworth Co. KS & 21.3 \\
\hline D1-I & Virgilian & Cass Ls. & Haskell Ls. & Woodson Co., KS & 19.9 \\
\hline D1-IV & Missourian & & Vinland Sh. & Woodson Co., KS & 19.9 \\
\hline C3-III & Missourian & & Vinland Sh. & Chautauqua Co., KS & 21.0 \\
\hline A6-XXI & Missourian & Stanton Ls. & Stoner Ls. & Wyandotte Co., KS & 21.0 \\
\hline$A 6-X X$ & Missourian & Stanton Ls. & Stoner Ls. & Wyandotte Co., KS & 21.0 \\
\hline P-15-3 & Missourian & Stanton Ls. & Stoner Ls. & Cass Co., IA & 21.7 \\
\hline P-15-2 & Missourian & Stanton Ls. & Stoner Ls. & Cass Co., IA & 21.3 \\
\hline P-15-1 & Missourian & Stanton Ls. & Stoner Ls. & Cass Co., IA & 21.0 \\
\hline $\mathrm{A} 6-\mathrm{XVI}$ & Missourian & Stanton Ls. & Eudora Sh. & Wyandotte Co., KS & 19.8 \\
\hline D3-I & Missourian & Stanton Ls. & Eudora Sh. & Anderson Co., KS & 19.7 \\
\hline D3-II & Missourian & Stanton Ls. & Eudora Sh. & Anderson Co., KS & 20.3 \\
\hline D3-III & Missourian & Stanton Ls. & Eudora Sh. & Anderson Co., KS & 20.3 \\
\hline He-15-3 (H) & Missourian & Stanton Ls. & Eudora Sh. & Cass Co., IA & 21.1 \\
\hline He-15-3 (S+I) & Missourian & Stanton Ls. & Eudora Sh. & Cass Co., IA & 21.0 \\
\hline $\mathrm{He}-15-2 \mathrm{~b}(\mathrm{H})$ & Missourian & Stanton Ls. & Eudora Sh. & Cass Co., IA & 20.3 \\
\hline $\mathrm{He}-15-2 b$ & Missourian & Stanton Ls. & Eudora Sh. & Cass Co., IA & 20.3 \\
\hline $\mathrm{He}-15-2(\mathrm{G})$ & Missourian & Stanton Ls. & Eudora Sh. & Cass Co., IA & 20.9 \\
\hline He-15-2 & Missourian & Stanton Ls. & Eudora Sh. & Cass Co., IA & 20.8 \\
\hline He-15-1 & Missourian & Stanton Ls. & Eudora Sh. & Cass Co., IA & 21.0 \\
\hline Le-15-1 & Missourian & Stanton Ls. & Captain Creek Ls. & Cass Co., IA & 21.2 \\
\hline$A 6-V I$ & Missourian & Plattsburg Ls & Spring Hill Ls. & Wyandotte Co., KS & 21.2 \\
\hline A6-V & Missourian & Plattsburg Ls & Spring Hill Ls. & Wyandotte Co., KS & 20.5 \\
\hline A6-IV & Missourian & Plattsburg Ls & Spring Hill Ls. & Wyandotte Co., KS & 20.6 \\
\hline A6-II & Missourian & Plattsburg Ls & Merriam Ls. & Wyandotte Co., KS & 21.0 \\
\hline A6-I & Missourian & Plattsburg Ls & Merriam Ls. & Wyandotte Co., KS & 22.2 \\
\hline A5-I & Missourian & Wvandotte Ls & Quindaro Sh. & Johnson Co., KS & 21.0 \\
\hline A4-IV & Missourian & Iola Ls. & Raytown Ls. & Wyandotte Co., KS & 21.5 \\
\hline A4-III & Missourian & Iola Ls. & Raytown Ls. & Wyandotte Co., KS & 21.1 \\
\hline A4-II & Missourian & Iola Ls. & Muncie Creek Sh. & Wyandotte Co., KS & 20.0 \\
\hline D4-I & Missourian & Iola Ls. & Muncie Creek Sh. & Woodson Co., KS & 20.7 \\
\hline A4-VI & Missourian & Iola Ls. & Paola Ls. & Wyandotte Co., KS & 20.9 \\
\hline A4-I & Missourian & Dewey Ls. & Quivira Sh. & Wyandotte Co., KS & 20.0 \\
\hline A3-III & Missourian & Dennis Ls. & Winterset Ls. & Jackson Co., KS & 21.2 \\
\hline A3-I & Missourian & Dennis Ls. & Stark Sh. & Jackson Co., KS & 21.0 \\
\hline A2-V & Missourian & Swope Ls. & Bethany Falls Ls. & Jackson Co., KS & 21.3 \\
\hline A2-IV & Missourian & Swope Ls. & Hushpuckney Sh. & Jackson Co., KS & 21.3 \\
\hline A2-II & Missourian & Swope Ls. & Hushpuckney Sh. & Jackson Co., KS & 19.9 \\
\hline A2-III & Missourian & Swope Ls. & Middle Creek Ls. & Jackson Co., KS & 21.8 \\
\hline A2-I & Missourian & Hertha Ls. & Mound City Sh. & Jackson Co., KS & 20.1 \\
\hline
\end{tabular}




\begin{tabular}{|c|c|c|c|c|c|}
\hline B1-IV & Missourian & Hertha Ls. & Mound City Sh. & Bourbon Co., KS & 18.9 \\
\hline B1-III & Missourian & Shale Hill Fm. & Critzler Ls. & Bourbon Co., KS & 20.9 \\
\hline B1-I & Missourian & Shale Hill Fm. & Exline Ls. & Bourbon Co., KS & 19.8 \\
\hline B8-1 & Missourian & Shale Hill Fm. & Exline Ls. & Nowata Co., OK & 20.0 \\
\hline A1-I & Desmoinesian & Lost Branch Fm. & Nuyaka Creek Sh. & Jackson Co., KS & 21.1 \\
\hline B4-I & Desmoinesian & Lenepah Ls. & Norfleet Ls. & Labette Co., KS & 20.6 \\
\hline B3-II & Desmoinesian & Altamont Ls. & Lake Neosho Sh. & Neosho Co., KS & 20.4 \\
\hline B3-I & Desmoinesian & Altamont Ls. & Lake Neosho Sh. & Neosho Co., KS & 20.1 \\
\hline B2-IV & Desmoinesian & Bandera Sh. & Fairlington Ls. & Crawford Co., KS & 20.3 \\
\hline B2-IIIb & Desmoinesian & Pawnee Ls. & Mine Creek Sh. & Crawford Co., KS & 20.4 \\
\hline B2-IIIa & Desmoinesian & Pawnee Ls. & Mine Creek Sh. & Crawford Co., KS & 20.6 \\
\hline B2-II & Desmoinesian & Pawnee Ls. & Myrick Station Ls. & Crawford Co., KS & 21.2 \\
\hline B2-I & Desmoinesian & Pawnee Ls. & Anna Sh. & Crawford Co., KS & 20.0 \\
\hline B7-I & Desmoinesian & Fort Scott Ls. & Higginsville Ls. & Labette Co., KS & 20.9 \\
\hline B7-II & Desmoinesian & Fort Scott Ls. & Higginsville Ls. & Labette Co., KS & 20.6 \\
\hline B7-III & Desmoinesian & Fort Scott Ls. & Little Osage Sh. & Labette Co., KS & 20.8 \\
\hline B7-VIII & Desmoinesian & Fort Scott Ls. & Excello Sh. & Labette Co., KS & 20.0 \\
\hline B7-VII & Desmoinesian & Fort Scott Ls. & Excello Sh. & Labette Co., KS & 20.0 \\
\hline B7-X & Desmoinesian & Fort Scott Ls. & Excello Sh. & Labette Co., KS & 19.4 \\
\hline B6-VII & Desmoinesian & Verdigris Ls. & Ardmore Ls. & Labette Co., KS & 21.4 \\
\hline $\mathrm{B} 6-\mathrm{VI}$ & Desmoinesian & Verdigris Ls. & Oakley Sh. & Labette Co., KS & 20.7 \\
\hline B6-IV & Desmoinesian & Verdigris Ls. & Oakley Sh. & Labette Co., KS & 21.2 \\
\hline B6-III & Desmoinesian & Verdigris Ls. & Oakley Sh. & Labette Co., KS & 21.2 \\
\hline B6-II & Desmoinesian & Verdigris Ls. & Oakley Sh. & Labette Co., KS & 19.7 \\
\hline
\end{tabular}

